\title{
Validation of the PI-RADS language: predictive values of PI-RADS lexicon descriptors for detection of prostate cancer
}

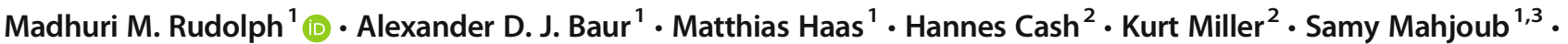 \\ Alexander Hartenstein ${ }^{1}$. David Kaufmann ${ }^{1}$. Roman Rotzinger ${ }^{1}$. Chau Hung Lee ${ }^{4}$. Patrick Asbach ${ }^{1} \cdot$ Bernd Hamm $^{1}$. \\ Tobias Penzkofer ${ }^{1,5}$
}

Received: 27 October 2019 / Revised: 22 January 2020 / Accepted: 21 February 2020 / Published online: 26 March 2020

(C) The Author(s) 2020

\begin{abstract}
Objectives To assess the discriminatory power of lexicon terms used in PI-RADS version 2 to describe MRI features of prostate lesions. Methods Four hundred fifty-four patients were included in this retrospective, institutional review board-approved study. Patients received multiparametric (mp) MRI and subsequent prostate biopsy including MRI/transrectal ultrasound fusion biopsy and 10core systematic biopsy. PI-RADS lexicon terms describing lesion characteristics on mpMRI were assigned to lesions by experienced readers. Positive and negative predictive values (PPV, NPV) of each lexicon term were assessed using biopsy results as a reference standard.

Results From a total of 501 lesions, clinically significant prostate cancer (csPCa) was present in 175 lesions (34.9\%). Terms related to findings of restricted diffusion showed PPVs of up to 52.0\%/43.9\% and NPV of up to $91.8 \% / 89.7 \%$ (peripheral zone or PZ/ transition zone or TZ). T2-weighted imaging (T2W)-related terms showed a wide range of predictive values. For PZ lesions, high PPVs were found for "markedly hypointense," "lenticular," "lobulated," and "spiculated" (PPVs between 67.2 and 56.7\%). For TZ lesions, high PPVs were found for "water-drop-shaped" and "erased charcoal sign" (78.6\% and 61.0\%). The terms "encapsulated," "organized chaos," and "linear" showed to be good predictors for benignity with distinctively low PPVs between 5.4 and 6.9\%. Most T2WI-related terms showed improved predictive values for TZ lesions when combined with DWI-related findings.

Conclusions Lexicon terms with high discriminatory power were identified (e.g., "markedly hypointense," "water-drop-shaped," "organized chaos"). DWI-related terms can be useful for excluding TZ cancer. Combining T2WI- with DWI findings in TZ lesions markedly improved predictive values.

Key Points

- Lexicon terms describing morphological and functional features of prostate lesions on MRI show a wide range of predictive values for prostate cancer.

- Some T2-related terms have favorable PPVs, e.g., "water-drop-shaped" and "organized chaos" while others show less distinctive predictive values. DWI-related terms have noticeable negative predictive values in TZ lesions making DWI feature a useful tool for exclusion of TZ cancer.
\end{abstract}

Electronic supplementary material The online version of this article (https://doi.org/10.1007/s00330-020-06773-1) contains supplementary material, which is available to authorized users.

Madhuri M. Rudolph

Madhuri.rudolph@charite.de

1 Department of Radiology, Freie Universität Berlin,

Humboldt-Universität zu Berlin and Berlin Institute of Health, Charité-Universitätsmedizin Berlin, Augustenburger Platz 1, 13353 Berlin, Germany

2 Department of Urology, Freie Universität Berlin,

Humboldt-Universität zu Berlin and Berlin Institute of Health, Charité-Universitätsmedizin Berlin, Charitéplatz 1, 13353 Berlin, Germany

3 Department of Urology, Universität zu Köln, Uniklinik Köln, Kerpener Str. 62, 50937 Köln, Germany

4 Department of Radiology, Tan Tock Seng Hospital, Singapore, Singapore

5 Berlin Institute of Health (BIH), Anna-Louisa-Karsch-Str. 2, 10178 Berlin, Germany 
- Combining DWI- and T2-related lexicon terms for assessment of TZ lesions markedly improves PPVs. Most T2-related lexicon terms showed a significant decrease in PPV when combined with negative findings for "DW hyperintensity."

Keywords Magnetic resonance imaging $\cdot$ Prostatic neoplasms $\cdot$ Predictive value of tests $\cdot$ Biopsy

$\begin{array}{ll}\text { Abbreviations } \\ \text { AS } & \text { Anterior stroma } \\ \text { csPCa } & \text { Clinically significant prostate cancer } \\ \text { CZ } & \text { Central zone } \\ \text { DCE } & \text { Dynamic contrast-enhanced } \\ \text { DWI } & \text { Diffusion-weighted imaging } \\ \text { GS } & \text { Gleason score } \\ \text { mpMRI } & \text { Multiparametric MRI } \\ \text { NPV } & \text { Negative predictive value } \\ \text { PCa } & \text { Prostate cancer } \\ \text { PI-RADS } & \text { Prostate Imaging Reporting and Data System } \\ \text { PPV } & \text { Positive predictive value } \\ \text { PZ } & \text { Peripheral zone } \\ \text { T2WI } & \text { T2-weighted } \\ \text { TB } & \text { Targeted MRI/TRUS fusion biopsy } \\ \text { TZ } & \text { Transition zone } \\ \text { v2 } & \text { Version 2 }\end{array}$

\section{Introduction}

Multiparametric magnetic resonance imaging (mpMRI) has emerged as a vital tool in the diagnosis of prostate cancer (PCa). Along with the widespread adoption of prostate MRI, a standardized interpretation and reporting of mpMRI findings have become necessary $[1,2]$. For this purpose, the Prostate Imaging Reporting and Data System (PI-RADS) was developed, based on a synthesis of expert consensus and available evidence. The revised PI-RADS version 2 (v2) was released in December 2014 [3]. Recently, in March 2019, modifications to PIRADS v2 have been published constituting an updated version termed PI-RADS v2.1 [4]. As PI-RADS is intended to be a document in evolution, studies have been encouraged to test its efficacy.

Despite recent developments in improving quantitative radiological methods, the vast majority of prostate cancer diagnosis on MRI is performed in the traditional "radiologist reporting" setting. Thus, the vocabulary and subjective assessments of the radiologist are the cornerstones of the reports' validity.

PI-RADS scoring is done by assessing lesions' features on T2-weighted (T2WI), diffusion-weighted (DWI), and dynamic contrast-enhanced imaging (DCE). The assessed criteria include lesions' signal intensity, shape, margins, size, and invasive behavior/extraprostatic extension. The PI-RADS v2 document provides a lexicon with defined descriptors in its appendix (Appendix III) that constitutes the very foundation of this assessment [3]. Analyzing and understanding this very foundation of PI-RADS could enable us to identify descriptors with high diagnostic accuracy, thus allowing these to be incorporated more prominently into the scoring criteria, while reducing the importance of descriptors with low accuracy.

Lexicon terms and their definitions remain largely unchanged in PI-RADS version 2.1, with the only changes being the redefinition of the term "negative DCE," a new definition of the term "marked" and the introduction of the terms "typical" and "atypical BPH nodule."

A number of studies have assessed the diagnostic value of the PI-RADS score [5-9], but to date, only sparse data is available concerning the underlying terminology. To the best of our knowledge, only few studies with small study populations have addressed the discriminatory power of individual lexicon terms $[10,11]$. Therefore, the objective of this study is to systematically assess the diagnostic value of individual descriptors as specified in the PI-RADS v2 lexicon in a large patient cohort.

\section{Materials and methods}

\section{Patients}

The inclusion criteria for this retrospective study were the availability of a prostate MRI between January 2012 and July 2015 and subsequent in-house targeted MRI/TRUS fusion biopsy (TB) in combination with a 10-core systematic biopsy in the same session. From a total of 526 eligible patients, 72 patients with incomplete or non-standard MRI or MRI performed at an external institution were excluded. These exclusions left a final cohort of 454 patients. Patient characteristics are summarized in Table 1. Figure 1 contains a STARD 2015-compliant patient flow diagram [12] for the study. The study protocol was approved by the institutional review board and patient consent was waived due to the retrospective design of the study. Subgroups of the same collective with various study endpoints have been included in earlier publications pertaining to the accuracy of prostate biopsies [13-18].

\section{MR imaging}

All imaging was performed on one of two identical 3-T MRI scanners (Skyra, Siemens Healthineers). The following imaging parameters were used in all patients: axial and coronal 
Table 1 Patient characteristics. Values are given as mean \pm standard deviation [range] for continuous variables and absolute frequency (relative frequency) for biopsy results. PSA, prostate-specific antigen; $P I N$, prostatic intraepithelial neoplasia

\begin{tabular}{lc}
\hline Parameter & Value \\
\hline Number of patients & 454 \\
Age (years) & $66.58 \pm 7.76[38-85]$ \\
PSA (ng/ml) & $13.17 \pm 15.49[0.65-199]$ \\
PSA density $\left(\mathrm{ng} / \mathrm{ml}^{2}\right)$ & $0.26 \pm 0.35[0.02-3.78]$ \\
Prostate volume $(\mathrm{ml})$ & $61.81 \pm 30.82[6.07-193.63]$ \\
Biopsy result (highest grade) & \\
No cancer nor inflammation & $65(14.3 \%)$ \\
Inflammation (chronic or acute) & $86(18.9 \%)$ \\
PIN & $12(2.6 \%)$ \\
$3+3$ & $91(20.0 \%)$ \\
$3+4$ & $65(14.3 \%)$ \\
$4+3$ & $42(9.3 \%)$ \\
$4+4$ & $71(15.6 \%)$ \\
$4+5$ & $15(3.3 \%)$ \\
$5+4$ & $4(0.9 \%)$ \\
$5+5$ & $3(0.7 \%)$ \\
\hline
\end{tabular}

T2WI with a resolution of $3.0 \times 0.47 \times 0.47 \mathrm{~mm}$, axial DWI with a resolution of $3 \times 1.4 \times 1.4 \mathrm{~mm}$ with measured $b$ values of 0,50 , and 500 and high $b$ value $(800,1000$, or a calculated $b$ value of $1400 \mathrm{~s} / \mathrm{mm}^{2}$ ), and additional T1 axial and T2 axial and sagittal imaging of the whole pelvis. In 242 patients (54.6\%), DCE imaging was performed additionally with a spatial resolution of $3 \times 1.4 \times 1.4 \mathrm{~mm}$, a temporal resolution of $5 \mathrm{~s}$, and a $3 \mathrm{ml} / \mathrm{s}$ injection flow (Gadobutrol, Gadovist, Bayer Healthcare).

\section{Imaging review and lexicon term assessment process}

Four hundred fifty-four MRI imaging datasets were divided into four similarly sized subgroups (113-114 each). Each group was evaluated by one of four readers (A.B., M.H., C.L., P.A.), all board-certified radiologists with more than 5 years of experience in prostate MRI. Each lesion was assessed once by a single reader using a dedicated in-house built reading software. The software presents all imaging in a standardized way to the reader (Fig. 2). Readers were blinded to all patient-related data including the initial radiological report and histopathological results. Readers were instructed to mark the most suspicious lesion or lesions in an MRI and tag every marked lesion with matching lexicon terms complying with the definitions supplied by the PI-RADS v2 lexicon. Definitions of all lexicon terms were displayed in the reading software exactly as specified in the lexicon of the original PIRADS v2 document [3]. Table 2 contains a full list of the used terms and their classification. All groupings of lexicon terms
(DWI-related, shape-related, border-related terms, etc.) were tagged separately. Lesions were attributed to either the peripheral zone (PZ) or the transition zone (TZ) and localized according to the segmentation model used in PI-RADS v2 [3]. Lesions that extended through $\mathrm{PZ}$ as well as $\mathrm{TZ}$ and lesions that were located in the anterior stroma (AS) or central zone (CZ) were assigned to either the PZ or the TZ group depending on the most probable zone of origin.

\section{Reference standard}

Prostate biopsies taken ahead of this study were performed by experienced urologists or interventional radiologists using one of two biopsy devices (Aplio 500, Toshiba or HI VISION Preirus, Hitachi Medical Systems) and consisted of TB and systematic 10-core biopsy. These were used as a reference standard. Histopathological findings were classified according to the Gleason grading system [19]. A Gleason score (GS) of $3+4$ or higher on TB or in a matching segment on systematic biopsy was considered a positive finding for clinically significant prostate cancer ( $\mathrm{cs} P C a)$. Tumor size or volume was not taken into consideration since size analysis was outside the scope of this study. Histopathological findings that indicated no cancerous changes (no tumor cells, acute prostatitis, chronic prostatitis, prostatic intraepithelial neoplasia, or benign prostatic hyperplasia) and GS $3+3$ tumor were considered non-csPCA.

\section{Statistical evaluation}

Positive and negative predictive values (PPVs, NPVs) as well as sensitivity and specificity for detection of csPCa were computed for each of the terms within each zone. For TZ lesions, PPVs of shape and border terms in combination with DWI/ADC terms were additionally analyzed. PPVs of term combinations were compared with PPVs of single terms using the generalized score test by Leisenring, Alonzo, and Pepe [20]. Results were declared to be significant if $p<0.05$. Statistical evaluation was performed using $\mathrm{R}$ version 1.1.419 (www.r-project.org) and Microsoft Excel version 16.16.17.

\section{Results}

\section{Lesion characteristics}

The readers marked 515 MRI lesions in the 454 MRI datasets. In 5 patients, no lesions were marked by the readers. Thirteen lesions were excluded due to unclear documentation of biopsy locations and one lesion was excluded due to its location in the seminal vesicles. This left a total of 501 lesions in 443 patients. CsPCa was detected in 175 (34.9\%) of the lesions. As 
Fig. 1 Flow chart with inclusion and exclusion criteria, as well as lesion localization and histopathological outcomes. All men received prostate biopsy including MRI/TRUS fusionguided targeted biopsy and systematic 10-core biopsy. mpMRI, multiparametric magnetic resonance imaging.; MRI/TRUS, MRI/transrectal ultrasound; GS, Gleason score
Subject Identification:

All consecutive patients with in-house MRI/TRUS fusion-guided targeted biopsy and systematic 10-core biopsy from 1/2012 - 7/2015 $(n=526)$

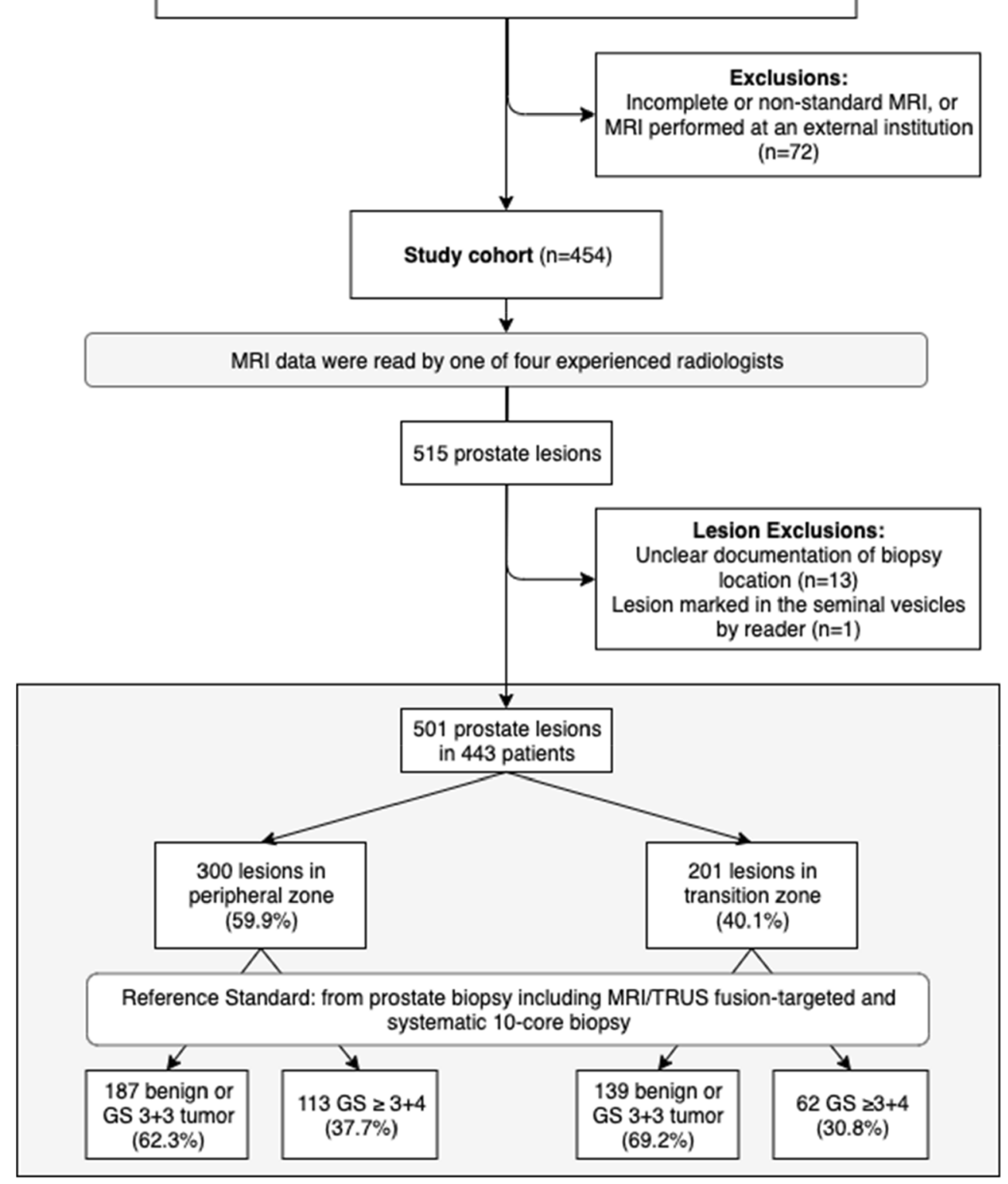

shown in Fig. 1, 300 (59.9\%) lesions were found in the PZ; prevalence of csPCa in $\mathrm{PZ}$ lesions was 113 (37.7\%). Two hundred one lesions $(40.1 \%)$ were located in the TZ; prevalence of csPCa in TZ lesions was 62 (30.8\%).

\section{Diagnostic performance of lexicon terms for PZ lesions}

Table 2 and Fig. 3a show PPVs and NPVs of the analyzed lexicon terms. Sensitivity and specificity are presented in Table 4 in the supplementary material. Lexicon terms with the highest PPVs were the following: restricted diffusion
(50.7\% [104/205]), DW hyperintensity (52.0\% [105/202]), early phase washin $(56.5 \%$ [48/85]), washout delayed phase (68.2\% [30/44]), positive DCE (55.4\% [56/101]), T2W markedly hypointense $(67.2 \%$ [43/64]), spiculated $(56.7 \%$ [17/ $30])$, lenticular (58.6\% [17/29]), lobulated (56.8\% [25/44]), water-drop-shaped (100.0\% [6/6]), and invasion (76.4\% [42/ 55]). Lexicon terms with the highest NPVs were the following: restricted diffusion (90.5\% [86/95]), DW hyperintensity (91.8\% [90/98]), and ADC hypointense (89.4\% [42/47]). Terms indicating benignity showed low PPVs for detection of csPCa, with the term "linear" displaying the lowest PPV of $6.3 \%(1 / 16)$. 


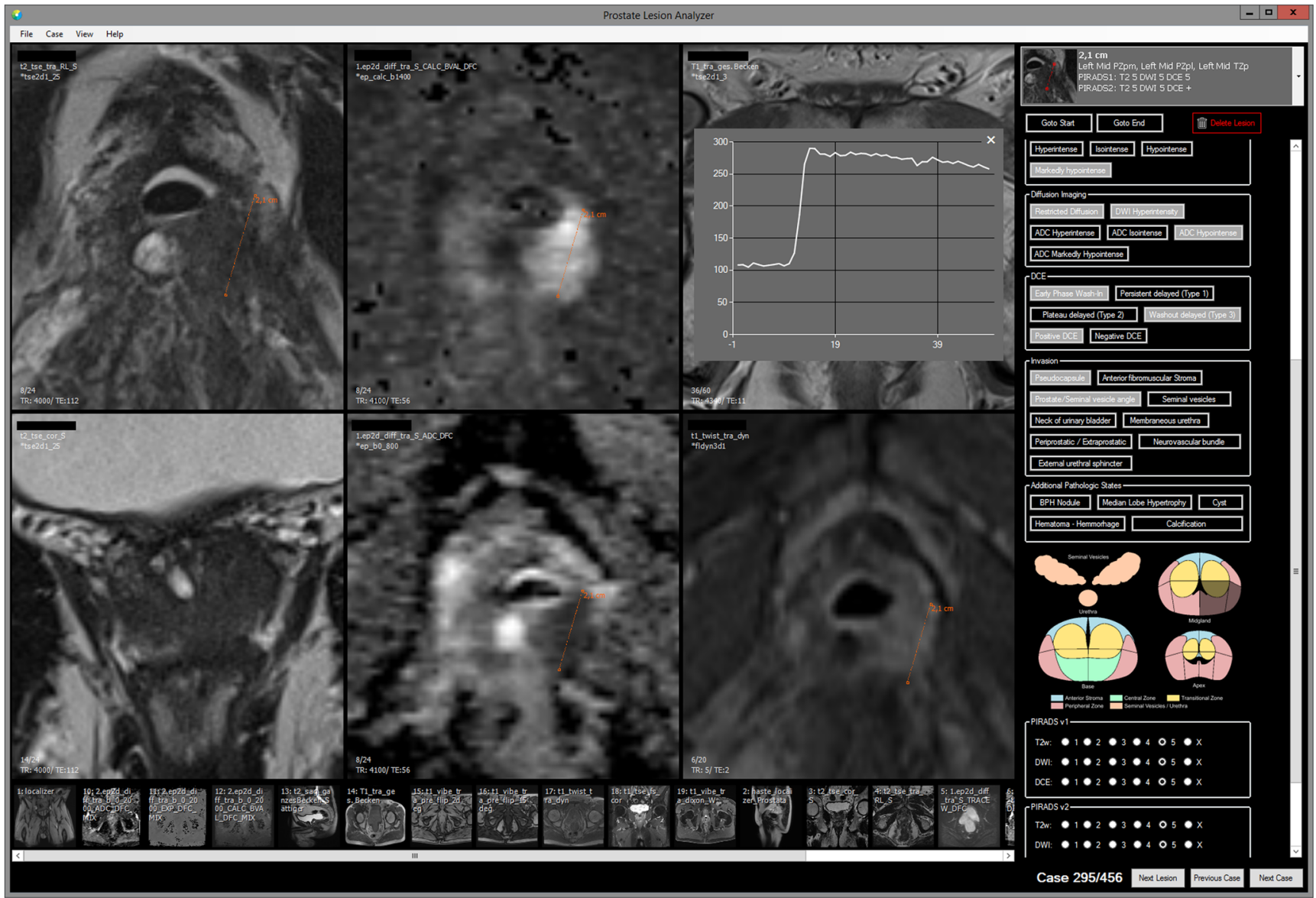

Fig. 2 Sample screenshot of the proprietary review software Prostate Lesion Analyzer used in the study. Readers were instructed to mark lesions within the MRI pictures (left, $3 \times 2$ panels depicting T2WI axial, T2WI coronal, DWI/ADC, T1WI native, and DCE). Matching lexicon terms and lesion localization were selected on the panel on the right side.

In a few cases, the readers also tagged PZ lesions with lexicon terms that are designed to describe TZ lesions (e.g., "erased charcoal sign" and "organized chaos"); these results are given in Table 2 and supplementary Table 4 for the sake of completeness.

\section{Diagnostic performance of lexicon terms for TZ lesions}

Table 2 and Fig. $3 b$ show predictive values of lexicon terms used for TZ lesions. The highest PPVs were found for the following lexicon terms: T2W markedly hypointense $(52.5 \%$ [21/40]), non-circumscribed (53.3\% [16/30]), irregular border $(50.0 \%$ [30/60]), spiculated $(57.1 \%$ [8/14]), erased charcoal sign $(61.0 \%$ [36/59]), water-drop-shaped $(78.6 \%$ [11/14]), and invasion $(58.9 \%$ [33/56]). Lexicon terms with the highest NPVs were the following: restricted diffusion $(85.1 \%$ [63/ 74]), DW hyperintensity (89.7\% [70/78]), and ADC hypointense $(87.0 \%$ [47/54]). Terms with the lowest PPVs were the following: T2W hyperintense $(3.1 \%[1 / 32])$, T2W
Definitions of lexicon terms were displayed exactly as specified in the original PI-RADS version 2 document when hovering the cursor over a term. T2WI, T2-weighted imaging; DWI/ADC, diffusion-weighted imaging/apparent diffusion coefficient; T1WI, T1-weighted imaging; DCE, dynamic contrast-enhanced imaging

isointense (5.3\% [1/19]), encapsulated (6.9\% [4/58]), and organized chaos $(5.4 \%$ [3/56]). Figure $3 \mathrm{c}$ summarizes predictive values of the most relevant lexicon terms that are currently used as assessment criteria in PI-RADS v2.1.

\section{PPVs of border and shape terms combined with diffusion-related terms used for TZ lesions}

Combining shape and border terms with the term "diffusionweighted hyperintensity" yielded the most distinctive PPVs for TZ lesions. PPVs of these term combinations are shown in Table 3. In most cases, combining a positive finding of "DW hyperintensity" with a shape- or border-related term increased the PPV mildly compared with the border/shape term alone, but this increase was of no statistical significance in most cases. On the other hand, combining border/shape terms with a negative finding for "DW hyperintensity" yielded significant changes in PPV, mostly. The following terms showed a statistically significant decrease of PPV when combined with a negative finding for "DW hyperintensity": 
Table 2 Predictive values of lexicon terms used for lesions in the PZ and TZ. Values are given as PPV in percent (cancer-positive lesions with term marked/all lesions with term marked) and NPV in percent (cancernegative lesions without term marked/all lesions without term marked).
$P Z$, peripheral zone; $T Z$, transition zone; $P P V$, positive predictive value; $N P V$, negative predictive value; $T P$, true positives; $D W I / A D C$, diffusionweighted imaging/apparent diffusion coefficient; $D C E$, dynamic contrastenhanced imaging; $D P$, delayed phase

\begin{tabular}{|c|c|c|c|c|c|}
\hline & \multirow[t]{2}{*}{ Terms } & \multicolumn{2}{|l|}{ Peripheral zone } & \multicolumn{2}{|l|}{ Transition zone } \\
\hline & & $\mathrm{PPV}$ in $\%$ & NPV in \% & PPV in $\%$ & NPV in $\%$ \\
\hline \multirow[t]{5}{*}{ DWI/ADC features } & Restricted diffusion & $50.7 \%(104 / 205)$ & $90.5 \%(86 / 95)$ & $40.2 \%(51 / 127)$ & $85.1 \%(63 / 74)$ \\
\hline & DW hyperintensity & $52.0 \%(105 / 202)$ & $91.8 \%(90 / 98)$ & $43.9 \%(54 / 123)$ & $89.7 \%(70 / 78)$ \\
\hline & ADC hyperintense & $0.0 \%(0 / 3)$ & $62.0 \%(184 / 297)$ & $0.0 \%(0 / 3)$ & $68.7 \%(136 / 198)$ \\
\hline & $\mathrm{ADC}$ isointense & $12.8 \%(5 / 39)$ & $58.6 \%(153 / 261)$ & $9.8 \%(4 / 41)$ & $63.8 \%(102 / 160)$ \\
\hline & ADC hypointense & $42.7 \%(108 / 253)$ & $89.4 \%(42 / 47)$ & $37.4 \%(55 / 147)$ & $87.0 \%(47 / 54)$ \\
\hline \multirow[t]{6}{*}{ DCE features* } & Early phase washin & $56.5 \%(48 / 85)$ & $75.8 \%(69 / 91)$ & $18.4 \%(7 / 38)$ & $70.8 \%(46 / 65)$ \\
\hline & Persistent DP (type 1) & $20.0 \%(10 / 50)$ & $52.4 \%(66 / 126)$ & $26.9 \%(7 / 26)$ & $75.3 \%(58 / 77)$ \\
\hline & Plateau DP (type 2) & $38.6 \%(27 / 70)$ & $59.4 \%(63 / 106)$ & $25.7 \%(9 / 35)$ & $75.0 \%(51 / 68)$ \\
\hline & Washout DP (type 3) & $68.2 \%(30 / 44)$ & $69.7 \%(92 / 132)$ & $25.8 \%(8 / 31)$ & $75.0 \%(54 / 72)$ \\
\hline & Positive DCE & $55.4 \%(56 / 101)$ & $81.3 \%(61 / 75)$ & $28.3 \%(13 / 46)$ & $77.2 \%(44 / 57)$ \\
\hline & Negative DCE & $13.6 \%(8 / 59)$ & $47.0 \%(55 / 117)$ & $26.0 \%(13 / 50)$ & $75.5 \%(40 / 53)$ \\
\hline \multirow[t]{4}{*}{ T2WI features } & Hyperintense & $15.8 \%(3 / 19)$ & $60.9 \%(171 / 281)$ & $3.1 \%(1 / 32)$ & $63.9 \%(108 / 169)$ \\
\hline & Isointense & $11.1 \%(1 / 9)$ & $61.5 \%(179 / 291)$ & $5.3 \%(1 / 19)$ & $66.5 \%(121 / 182)$ \\
\hline & Hypointense & $34.9 \%(91 / 261)$ & $43.6 \%(17 / 39)$ & $29.8 \%(51 / 171)$ & $63.3 \%(19 / 30)$ \\
\hline & Markedly hypointense & $67.2 \%(43 / 64)$ & $70.3 \%(166 / 236)$ & $52.5 \%(21 / 40)$ & $74.5 \%(120 / 161)$ \\
\hline \multirow[t]{9}{*}{ Border } & Circumscribed & $41.0 \%(55 / 134)$ & $65.1 \%(108 / 166)$ & $20.4 \%(22 / 108)$ & $57.0 \%(53 / 93)$ \\
\hline & Non-circumscribed & $25.9 \%(14 / 54)$ & $59.8 \%(147 / 246)$ & $53.3 \%(16 / 30)$ & $73.1 \%(125 / 171)$ \\
\hline & Indistinct & $28.7 \%(37 / 129)$ & $55.6 \%(95 / 171)$ & $37.7 \%(26 / 69)$ & $72.7 \%(96 / 132)$ \\
\hline & Obscured & $37.6 \%(35 / 93)$ & $62.3 \%(129 / 207)$ & $32.8 \%(21 / 64)$ & $70.1 \%(96 / 137)$ \\
\hline & Irregular & $43.8 \%(46 / 105)$ & $65.6 \%(128 / 195)$ & $50.0 \%(30 / 60)$ & $77.3 \%(109 / 141)$ \\
\hline & Spiculated & $56.7 \%(17 / 30)$ & $64.4 \%(174 / 270)$ & $57.1 \%(8 / 14)$ & $71.1 \%(133 / 187)$ \\
\hline & Encapsulated & $11.1 \%(1 / 9)$ & $61.5 \%(179 / 291)$ & $6.9 \%(4 / 58)$ & $59.4 \%(85 / 143)$ \\
\hline & Organized chaos & $14.3 \%(1 / 7)$ & $61.8 \%(181 / 293)$ & $5.4 \%(3 / 56)$ & $59.3 \%(86 / 145)$ \\
\hline & Erased charcoal sign & $80.0 \%(8 / 10)$ & $63.8 \%(185 / 290)$ & $61.0 \%(36 / 59)$ & $81.7 \%(116 / 142)$ \\
\hline \multirow[t]{8}{*}{ Shape } & Round & $25.9 \%(14 / 54)$ & $59.8 \%(147 / 246)$ & $21.4 \%(9 / 42)$ & $66.7 \%(106 / 159)$ \\
\hline & Oval & $45.7 \%(43 / 94)$ & $66.0 \%(136 / 206)$ & $15.6 \%(12 / 77)$ & $59.7 \%(74 / 124)$ \\
\hline & Lenticular & $58.6 \%(17 / 29)$ & $64.6 \%(175 / 271)$ & $44.7 \%(17 / 38)$ & $72.4 \%(118 / 163)$ \\
\hline & Lobulated & $56.8 \%(25 / 44)$ & $65.6 \%(168 / 256)$ & $46.3 \%(19 / 41)$ & $73.1 \%(117 / 160)$ \\
\hline & Water-drop-shaped & $100.0 \%(6 / 6)$ & $63.6 \%(187 / 294)$ & $78.6 \%(11 / 14)$ & $72.7 \%(136 / 187)$ \\
\hline & Wedge-shaped & $20.0 \%(13 / 65)$ & $57.4 \%(135 / 235)$ & $50.0 \%(1 / 2)$ & $69.3 \%(138 / 199)$ \\
\hline & Linear & $6.3 \%(1 / 16)$ & $60.6 \%(172 / 284)$ & $-(0 / 0)$ & $69.2 \%(139 / 201)$ \\
\hline & Irregular & $38.7 \%(43 / 111)$ & $63.0 \%(119 / 189)$ & $39.3 \%(24 / 61)$ & $72.9 \%(102 / 140)$ \\
\hline Invasion & Invasion & $76.4 \%(42 / 55)$ & $71.0 \%(174 / 245)$ & $58.9 \%(33 / 56)$ & $80.0 \%(116 / 145)$ \\
\hline
\end{tabular}

*Only for patients with DCE imaging

circumscribed, non-circumscribed, indistinct, obscured, irregular border, round, oval, lenticular, lobulated, water-dropshaped, and irregular shape.

\section{Discussion}

In this study, the predictive power of PI-RADS v2 lexicon terms was analyzed with the aim of adding to the quantitative support of the PI-RADS guideline and identifying areas of improvement.

On the one hand, the presented data corroborates the use of many established assessment criteria in PI-RADS. Lexicon terms indicating a restricted diffusion in PZ lesions showed favorable combinations of both relatively high PPVs and high NPVs (e.g., PPV of $50.7 \%$ and NPV of $90.5 \%$ for the term "restricted diffusion"). Our work therefore confirms the importance of diffusion-related findings in the PZ [21-23]. 

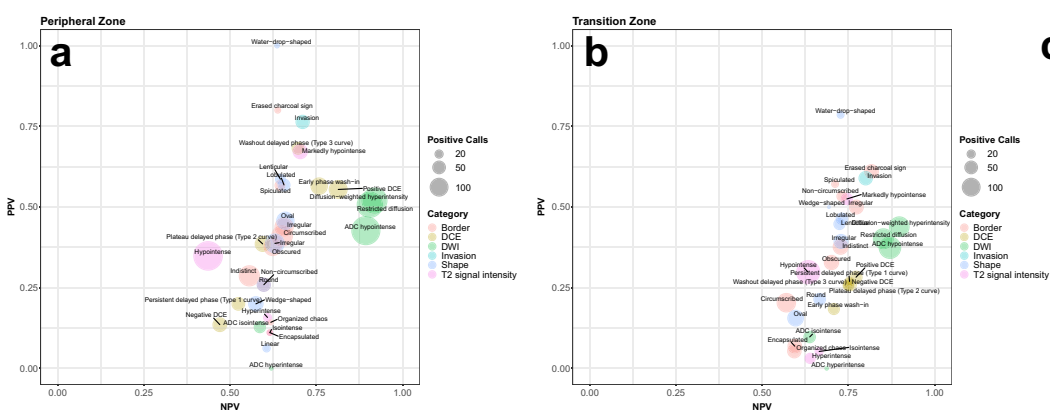

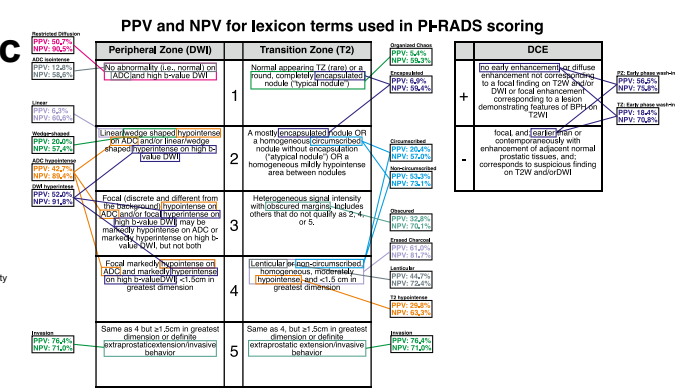

Fig. 3 Predictive values of PI-RADS v2 lexicon terms for peripheral zone (a) and transition zone (b). Current PI-RADS v2.1 assessment criteria with their respective predictive values as found in this study are shown in (c). PPV and NPV approximating 1 were considered favorable for terms indicating malignancy. PPV and NPV approximating 0 were considered favorable for terms indicating benignity. PPV, positive predictive value; $\mathrm{NPV}$, negative predictive value; DCE, dynamic contrast-enhanced imaging; DWI, diffusion-weighted imaging
Conflicting data have been published regarding the value of DCE imaging in cancer detection. The current consensus is that the addition of DCE imaging to DW imaging increases cancer detection in the PZ, while it might not be useful in $\mathrm{TZ}$ lesions [5, 24-27]. Our results support this consensus, with positive DCE findings showing high PPVs up to $68.2 \%$ in PZ lesions and poorer performance in TZ lesions with PPV at a maximum of $28.3 \%$. Moreover, signs of invasive behavior or extraprostatic extension are considered highly suggestive of cancer [3]. In accordance with that, our study showed high PPVs for the term "invasion" (76.4\% and $58.9 \%$ for PZ and $\mathrm{TZ}$ lesions, respectively). Features that are suggestive of
Table 3 Positive predictive values of border- and shape-related terms describing $\mathrm{TZ}$ lesions combined with positive or negative findings for diffusion-weighted hyperintensity. Values are given as PPV in percent (cancer-positive lesions with term(s) marked/all lesions with term(s) marked). PPVs of term combinations were compared with PPVs of single terms using the generalized score test by Leisenring, Alonzo, and Pepe
[20]. Differences in PPV were considered significant if $p$ values were below 0.05 , these values are set in italic. $T Z$, transition zone; $P P V$, positive predictive value; $N P V$, negative predictive value; $D W I$, diffusionweighted imaging; $T P$, true positives; $P O S$, all positives; $T N$, true negatives; $N E G$, all negatives

\begin{tabular}{|c|c|c|c|c|c|c|c|c|}
\hline \multirow[b]{2}{*}{ Feature group } & \multirow[b]{2}{*}{ Items } & \multirow{2}{*}{$\begin{array}{l}\text { Border/shape } \\
\text { term alone } \\
\text { PPV in \% } \\
\text { (TP/ POS) }\end{array}$} & \multicolumn{3}{|c|}{$\begin{array}{l}\text { Border/shape term combined with } \\
\text { positive finding for DW hyperintensity }\end{array}$} & \multicolumn{3}{|c|}{$\begin{array}{l}\text { Border/shape term combined with } \\
\text { negative finding for DW hyperintensity }\end{array}$} \\
\hline & & & $\begin{array}{l}\text { PPV in \% } \\
\text { (TP/ POS) }\end{array}$ & $\begin{array}{l}\text { Relative change } \\
\text { compared with } \\
\text { border/shape } \\
\text { term alone }\end{array}$ & $p$ value & $\begin{array}{l}\mathrm{PPV} \text { in \% } \\
\text { (TP/ POS) }\end{array}$ & $\begin{array}{l}\text { Relative Change } \\
\text { compared with } \\
\text { border/shape } \\
\text { term alone }\end{array}$ & $p$ value \\
\hline \multirow[t]{9}{*}{ Border } & Circumscribed & $20.4 \%(22 / 108)$ & $35.7 \%(20 / 56)$ & $+15.3 \%$ & 0.0013 & $3.8 \%(2 / 52)$ & $-16.5 \%$ & 0.0016 \\
\hline & Non-circumscribed & $53.3 \%(16 / 30)$ & $58.3 \%(14 / 24)$ & $+5.0 \%$ & 0.4015 & $33.3 \%(2 / 6)$ & $-20.0 \%$ & 0.0000 \\
\hline & Indistinct & $37.7 \%(26 / 69)$ & $43.4 \%(23 / 53)$ & $+5.7 \%$ & 0.1838 & $18.8 \%(3 / 16)$ & $-18.9 \%$ & 0.0000 \\
\hline & Obscured & $32.8 \%(21 / 64)$ & $41.5 \%(17 / 41)$ & $+8.7 \%$ & 0.0417 & $17.4 \%(4 / 23)$ & $-15.4 \%$ & 0.0000 \\
\hline & Irregular & $50.0 \%(30 / 60)$ & $54.0 \%(27 / 50)$ & $+4.0 \%$ & 0.3320 & $30.0 \%(3 / 10)$ & $-20.0 \%$ & 0.0000 \\
\hline & Spiculated & $57.1 \%(8 / 14)$ & $57.1 \%(8 / 14)$ & $0 \%$ & 1.0000 & $-(0 / 0)$ & - & NA \\
\hline & Encapsulated & $6.9 \%(4 / 58)$ & $16.7 \%(2 / 12)$ & $+9.8 \%$ & 0.0000 & $4.3 \%(2 / 46)$ & $-2.5 \%$ & 0.3721 \\
\hline & Organized chaos & $5.4 \%(3 / 56)$ & $12.5 \%(1 / 8)$ & $+7.1 \%$ & 0.0000 & $4.2 \%(2 / 48)$ & $-1.2 \%$ & 0.5539 \\
\hline & Erased charcoal sign & $61.0 \%(36 / 59)$ & $60.0 \%(33 / 55)$ & $-1.0 \%$ & 0.6767 & $75.0 \%(3 / 4)$ & $+14.0 \%$ & 0.0000 \\
\hline \multirow[t]{8}{*}{ Shape } & Round & $21.4 \%(9 / 42)$ & $35.0 \%(7 / 20)$ & $+13.6 \%$ & 0.0102 & $9.1 \%(2 / 22)$ & $-12.3 \%$ & 0.0290 \\
\hline & Oval & $15.6 \%(12 / 77)$ & $26.3 \%(10 / 38)$ & $+10.7 \%$ & 0.0031 & $5.1 \%(2 / 39)$ & $-10.5 \%$ & 0.0068 \\
\hline & Lenticular & $44.7 \%(17 / 38)$ & $45.7 \%(16 / 35)$ & $+1.0 \%$ & 0.7326 & $33.3 \%(1 / 3)$ & $-11.4 \%$ & 0.0000 \\
\hline & Lobulated & $46.3 \%(19 / 41)$ & $52.9 \%(18 / 34)$ & $+6.6 \%$ & 0.4198 & $14.3 \%(1 / 7)$ & $-32.1 \%$ & 0.0000 \\
\hline & Water-drop-shaped & $78.6 \%(11 / 14)$ & $83.3 \%(10 / 12)$ & $+4.8 \%$ & 0.6740 & $50.0 \%(1 / 2)$ & $-28.6 \%$ & 0.0011 \\
\hline & Wedge-shaped & $50.0 \%(1 / 2)$ & $50.0 \%(1 / 2)$ & $0 \%$ & 1.0000 & $-(0 / 0)$ & - & NA \\
\hline & Linear & $-(0 / 0)$ & $-(0 / 0)$ & - & NA & $-(0 / 0)$ & - & NA \\
\hline & Irregular & $39.3 \%(24 / 61)$ & $45.7 \%(21 / 46)$ & $+6.3 \%$ & 0.1725 & $20.0 \%(3 / 15)$ & $-19.3 \%$ & 0.0000 \\
\hline \multirow[t]{2}{*}{ DWI } & DW hyperintensity & $52.0 \%(105 / 202)$ & & & & & & \\
\hline & No DW hyperintensity & $10.3 \%(8 / 78)$ & & & & & & \\
\hline
\end{tabular}


benign findings, such as "encapsulated" and "organized chaos" for TZ lesions or "linear" for PZ lesions, were consistently associated with benign biopsy outcomes in this study.

On the other hand, our study showed areas of discriminatory potential that are currently not fully utilized in the PIRADS v2 and v2.1 assessment: In PI-RADS v2, diffusionrelated findings play a minor role for TZ scoring [3]. In PIRADS v2.1, however, the DWI score has gained more importance and scores of 4 and 5 can now upgrade the overall score of a TZ lesion [4]. This adjustment is consistent with studies demonstrating lower $\mathrm{ADC}$ values in $\mathrm{TZ}$ cancers than in $\mathrm{BPH}$ nodules, albeit with a large overlap of ADC values [26, 28-30], and studies showing higher diagnostic accuracy when T2WI- and DWI assessments were combined in TZ lesions $[11,31]$. The presented data shows another potential of DWI findings: the terms "restricted diffusion," "diffusion-weighted hyperintensity," and "ADC hypointense" had high NPVs of $89.4 \%$ to $91.8 \%$ for TZ lesions, whereas T2WI-related terms showed lower NPVs with a maximum of $66.0 \%$ for TZ lesions. Combining T2WI-related border and shape features with a finding of absent diffusion-weighted hyperintensity lowered the respective PPV markedly in most cases compared with the T2WI-related term alone. The means of integrating this negative predictive potential of DWI-related terms into the scoring system in addition to the positive predictive potential of T2WI-related findings could therefore be considered. From the data presented in this study, the absence of features related to restricted diffusion can be assumed to have a good potential to exclude csPCa in the TZ and could be used to downgrade $\mathrm{TZ}$ lesions.

Furthermore, we identified a number of border- and shaperelated terms with high PPVs, which are currently not explicitly included as criteria for PI-RADS v2 and v2.1 scores: For both PZ and TZ lesions, the descriptors "lenticular," "lobulated," and "spiculated" showed rather high PPVs between 44.7 and $58.6 \%$. In $\mathrm{TZ}$ lesions, the descriptors "water-dropshaped," "irregular," "non-circumscribed," and "erased charcoal sign" also showed high PPVs between 50.0 and 78.6\%. In a previous study with 14 included patients, Pokharel et al [11] found a similar PPV for TZ lesions with an irregular border (55\%). The term "organized chaos" showed a favorable PPV of $5.4 \%$ indicating benignity in $\mathrm{TZ}$ lesions. Including these highly discriminatory terms into the assessment criteria for PI-RADS categories should be considered.

There are a number of limitations to this study, potentially influencing the generalizability of the results. The standard of reference was histopathologic results of TB and a systematic 10-core biopsy taken ahead of the re-read conducted in this study. Although this method has been shown to have high rates of detection of malignant diseases [32], some malignant lesions may not have been targeted upon TB and may have been missed upon systematic biopsy. Rouvière et al [33] report in a large, prospective, multicenter study that GS $\geq 7$ tumor would have been missed in $7.6 \%$ (95\% CI, $4.6-$ $11.6 \%$ ) of patients, had TB not been done. The presence of false negatives could influence the reliability of diagnostic values referring to benign-appearing lesions especially, since these were not targeted upon TB. Furthermore, the fact that only patients that underwent biopsy were included may affect the results pertaining to benign-appearing lesions, as patients without suspicious lesions on MRI and low clinical risk factors did not undergo biopsy and were thus excluded from the study. Moreover, benign-appearing lesions in subjects with other suspicious areas may not have been identified as targets by the readers. A more reliable standard of reference would be histopathology after surgical prostatectomy, though this would bias the underlying collective towards mediumaggressive cancers.

Another limitation stems from interreader variability, which was not assessed in this study, as each MRI was read once by a single experienced reader. Additionally, three of the four readers worked at the same institution. The assignment of lexicon terms is, however, a subjective process. Several other studies have shown moderate interobserver agreement for PIRADS final assessment $[8,34]$ and few studies have also demonstrated moderate interobserver agreement for assignment of individual lexicon terms [10,34]. Nevertheless, larger multicenter studies that assess interobserver agreement in the assignment of lexicon descriptors could improve the generalizability of their predictive values.

\section{Conclusions}

The present study identifies lexicon terms with high and low discriminatory power for the prediction of csPCa. The presented data corroborate the importance of DWI/ADC- and DCErelated findings in the PZ by showing favorable PPVs and NPVs in the respective lexicon terms. We identify T2WIrelated terms with high PPVs: "markedly hypointense," "water drop-shaped," and "spiculated" for the PZ and TZ; "lobulated" for the PZ; and "erased charcoal sign", "noncircumscribed," and "irregular border" for the TZ. Moreover, this study demonstrates that DWI/ADC-related lexicon terms can be useful for excluding csPCa in the TZ. We show that combining T2WI-findings with findings of absent DW hyperintensity in TZ lesions significantly decreases PPVs. While with the new PI-RADS v2.1 the positive predictive potential of DWI-findings in TZ lesions has been more prominently utilized, means of incorporating the negative predictive potential of DWI-related terms for TZ lesions (e.g., by downgrading lesions) could further increase diagnostic accuracy.

Funding information Open Access funding provided by Projekt DEAL. The authors state that this work has not received any funding. 


\section{Compliance with ethical standards}

Guarantor The scientific guarantor of this publication is Mr. Tobias Penzkofer.

Conflict of interest The authors of this manuscript declare relationships with the following companies:

Dr. Penzkofer reports research agreements (no personal payments, outside of submitted work) with AGO, Aprea AB, ARCAGY-GINECO, Astellas Pharma Global Inc. (APGD), Astra Zeneca, Clovis Oncology, Inc., Dohme Corp, Holaira, Incyte Corporation, Karyopharm, Lion Biotechnologies, Inc., MedImmune, Merck Sharp, Millennium Pharmaceuticals, Inc., Morphotec Inc., NovoCure Ltd., PharmaMar S.A. and PharmaMar USA, Inc., Roche, Siemens Healthineers, TESARO Inc.

Prof. Hamm reports agreements (grant money to the Department of Radiology, Charite Berlin) with the following companies or non-profit organizations: Abbott, AbbVie, Ablative Solutions, Accovion, Achaogen Inc., Actelion Pharmaceuticals, ADIR, Aesculap, AGO, AIF Arbeitsgemeinschaft industrieller Forschungsvereinigungen, AIO: Arbeitsgemeinschaft Internistische Onkologie, Alexion Pharmaceuticals, Amgen, AO Foundation, Arena Pharmaceiticals, ARMO Biosciences, Inc., art photonics GmbH Berlin, ASR Advanced sleep research, Astellas, AstraZeneca, BARD, Bayer Healthcare, Bayer Schering Pharma, Bayer Vital, BBraun (Sponsoring eines Workshops), BerlinBrandenburger Centrum für Regenerative Therapien (BCRT), Berliner Krebsgesellschaft, Biotronik, Bioven, BMBF, Boehring Ingelheimer, Boston Biomedical Inc., BRACCO Group, Brainsgate, Bristol-Myers Squibb, Cascadian Therapeutics, Inc., Celgene, CELLACT Pharma, Celldex Therapeutics, CeloNova BioSciences, Charité research organisation $\mathrm{GmbH}$, Chiltern, CLOVIS ONCOLOGY, INC., Covance, CUBIST, CureVac AG, Tübingen, Curis, Daiichi, DC Devices, Inc. USA, Delcath Systems, Dermira Inc., Deutsche Krebshilfe, Deutsche Rheuma Liga, DFG, DSM Nutritional Products AG, Dt. Stiftung für Herzforschung, Dynavax, Eisai Ltd., European Knowledge Centre, Mosquito Way, Hatfield, Eli Lilly and Company Ltd., EORTC, Epizyme, Inc., Essex Pharma, EU Programmes, Euroscreen S.A., Fibrex Medical Inc., Focused Ultrasound Surgery Foundation, Fraunhofer Gesellschaft, Galena Biopharma, Galmed Research and Development Ltd., Ganymed, GE, Genentech. Inc., GETNE (Grupo Español de Tumores Neuroendocrinos), Gilead Sciences, Inc., Glaxo Smith Kline, Glycotope GmbH, Berlin, Goethe Uni Frankfurt, Guerbet, Guidant Europe NV, Halozyme, Hewlett Packard GmbH, Holaira Inc., ICON (CRO), Idera Pharmaceuticals, Inc., Ignyta, Inc., Immunomedics Inc., Immunocore, Incyte, INC Research, Innate Pharma, InSightec Ltd., Inspiremd, inVentiv Health Clinical UK Ltd., Inventivhealth, IOMEDICO, IONIS, IPSEN Pharma, IQVIA, ISA Therapeutics, Isis Pharmaceuticals Inc., ITM Solucin GmbH, Jansen, Kantar Health GmbH (CRO), Kartos Therapeutics, Inc., Karyopharm Therapeutics, Inc., Kendle/ MorphoSys AG, Kite Pharma, Kli Fo Berlin Mitte, La Roche, Land Berlin, Lilly $\mathrm{GmbH}$, Lion Biotechnology, Lombard Medical, Loxo Oncology, Inc., LSK BioPartners; USA, Lundbeck GmbH, LUX Biosciences, LYSARC, MacroGenics, MagForce, MedImmune Inc., MedImmune Limited, Medpac, Medpace Germany GmbH (CRO), MedPass (CRO), Medronic, Merck, Merrimack Pharmaceuticals Inc., MeVis Medical Solutions AG, Millennium Pharmaceuticals Inc., Mologen, Monika Kutzner Stiftung, MSD Sharp, NeoVacs SA, Newlink Genentics Corporation, Nexus Oncology, NIH, Novartis, Novocure, Nuvisan, Ockham oncology, OHIRC Kanada, Orion Corporation Orion Pharma, Parexel CRO Service, Perceptive, Pfizer GmbH, PharmaCept GmbH, Pharma Mar, Pharmaceutical Research Associates GmbH (PRA), Pharmacyclics Inc., Philipps, PIQUR Therapeutics Ltd., Pluristem, PneumRX. Inc., Portola Pharmaceuticals, PPD (CRO), PRAint, Premier-research, Provectus Biopharmaceuticals, Inc., psi-cro, Pulmonx International Sàrl, Quintiles Gmbh, Regeneron Pharmaceuticals, Inc., Respicardia, Roche, Samsung, Sanofi, sanofis-aventis S.A, Schumacher GmbH (Sponsoring eines
Workshops), Seattle Genetics, Servier (CRO), SGS Life Science Services (CRO), Shire Human Genetic Therapies, Siemens, Silena Therapeutics, Spectranetics GmbH, Spectrum Pharmaceuticals, St. Jude Medical, Stiftung Wolfgang Schulze, Symphogen, Taiho Oncology, Inc., Taiho Pharmaceutical Co., TauRx Therapeutics Ltd., Terumo Medical Corporation, Tesaro, tetec-ag, TEVA, Theorem, Theradex, Threshold Pharmaceuticals Inc., TNS Healthcare GMbH, Toshiba, UCB Pharma, Uni München, VDI / VDE, Vertex Pharmaceuticals Incorporated, winicker-norimed, Wyeth Pharma, Xcovery Holding Company, Zukunftsfond Berlin (TSB).

All other authors do not declare any conflicts of interest.

Statistics and biometry No complex statistical methods were necessary for this paper.

Informed consent Written informed consent was waived by the Institutional Review Board.

Ethical approval Institutional Review Board approval was obtained.

Study subjects or cohorts overlap Some study subjects or cohorts have been previously reported in the below listed articles. These studies had independent endpoints from this study. The scope of these studies was the accuracy of targeted and systematic prostate biopsies. Data about PIRADS lexicon terms in this cohort have not been published before.

Cash H, Gunzel K, Maxeiner A et al (2016) Prostate cancer detection on transrectal ultrasonography-guided random biopsy despite negative real-time magnetic resonance imaging/ultrasonography fusion-guided targeted biopsy: reasons for targeted biopsy failure. BJU Int 118:35-43.

Cash H, Maxeiner A, Stephan C et al (2016) The detection of significant prostate cancer is correlated with the Prostate Imaging Reporting and Data System (PI-RADS) in MRI/transrectal ultrasound fusion biopsy. World J Urol 34:525-532.

Günzel K, Cash H, Buckendahl J et al (2017) The addition of a sagittal image fusion improves the prostate cancer detection in a sensor-based MRI /ultrasound fusion guided targeted biopsy. BMC Urology 17:7.

Günzel K, Haas M, Maxeiner A et al (2017) Predictive parameters identifying men eligible for a sole MRI/ultrasound fusion-guided targeted biopsy without an additional systematic biopsy. Urologia Internationalis 98:15-21.

Maxeiner A, Kittner B, Blobel C et al (2018) Primary magnetic resonance imaging/ultrasonography fusion-guided biopsy of the prostate. BJU International 122:211-218.

Maxeiner A, Stephan C, Durmus T, Slowinski T, Cash H, Fischer T (2015) Added value of multiparametric ultrasonography in magnetic resonance imaging and ultrasonography fusion-guided biopsy of the prostate in patients with suspicion for prostate cancer. Urology 86:108-114.

\section{Methodology \\ - Retrospective \\ - Diagnostic or prognostic study \\ - Performed at one institution}

Open Access This article is licensed under a Creative Commons Attribution 4.0 International License, which permits use, sharing, adaptation, distribution and reproduction in any medium or format, as long as you give appropriate credit to the original author(s) and the source, provide a link to the Creative Commons licence, and indicate if changes were made. The images or other third party material in this article are included in the article's Creative Commons licence, unless indicated otherwise in a credit line to the material. If material is not included in the article's Creative Commons licence and your intended use is not permitted by statutory regulation or exceeds the permitted use, you will need to obtain permission directly from the copyright holder. To view a copy of this licence, visit http://creativecommons.org/licenses/by/4.0/. 


\section{References}

1. Kirkham AP, Emberton M, Allen C (2006) How good is MRI at detecting and characterising cancer within the prostate? Eur Urol 50:1163-1174 discussion 1175

2. Dickinson L, Ahmed HU, Allen C et al (2011) Magnetic resonance imaging for the detection, localisation, and characterisation of prostate cancer: recommendations from a European consensus meeting. Eur Urol 59:477

3. Weinreb JC, Barentsz JO, Choyke PL et al (2016) PI-RADS prostate imaging - reporting and data system: 2015, version 2. Eur Urol 69:16-40

4. Turkbey B, Rosenkrantz AB, Haider MA et al (2019) Prostate Imaging Reporting and Data System Version 2.1: 2019 Update of prostate imaging reporting and data system version 2. Eur Urol. https://doi.org/10.1016/j.eururo.2019.02.033

5. Greer MD, Shih JH, Lay N et al (2017) Validation of the dominant sequence paradigm and role of dynamic contrast-enhanced imaging in PI-RADS version 2. Radiology 285:859-869

6. Rosenkrantz AB, Babb JS, Taneja SS, Ream JM (2017) Proposed adjustments to PI-RADS version 2 decision rules: impact on prostate cancer detection. Radiology 283:119-129

7. Giannarini G, Girometti R, Crestani A et al (2019) A prospective accuracy study of prostate imaging reporting and data system version 2 on multiparametric magnetic resonance imaging in detecting clinically significant prostate cancer with whole-mount pathology. Urology 123:191-197

8. Muller BG, Shih JH, Sankineni S et al (2015) Prostate cancer: interobserver agreement and accuracy with the revised prostate imaging reporting and data system at multiparametric MR imaging. Radiology 277:741-750

9. Baldisserotto M, Neto EJ, Carvalhal G et al (2016) Validation of PIRADS v.2 for prostate cancer diagnosis with MRI at 3T using an external phased-array coil. J Magn Reson Imaging 44:1354-1359

10. Benndorf M, Hahn F, Kronig M et al (2017) Diagnostic performance and reproducibility of $\mathrm{T} 2 \mathrm{w}$ based and diffusion weighted imaging (DWI) based PI-RADSv2 lexicon descriptors for prostate MRI. Eur J Radiol 93:9-15

11. Pokharel SS, Patel NU, Garg K et al (2015) Multi-parametric MRI findings of transitional zone prostate cancers: correlation with 3dimensional transperineal mapping biopsy. Abdom Imaging 40: 143-150

12. Bossuyt PM, Reitsma JB, Bruns DE et al (2015) STARD 2015: an updated list of essential items for reporting diagnostic accuracy studies. Radiology 277:826-832

13. Cash H, Günzel K, Maxeiner A et al (2016) Prostate cancer detection on transrectal ultrasonography-guided random biopsy despite negative real-time magnetic resonance imaging/ultrasonography fusion-guided targeted biopsy: reasons for targeted biopsy failure. BJU Int 118(1):35-43

14. Cash H, Maxeiner A, Stephan C et al (2016) The detection of significant prostate cancer is correlated with the prostate imaging reporting and data system (PI-RADS) in MRI/transrectal ultrasound fusion biopsy. World J Urol 34(4):525-532

15. Günzel K, Cash H, Buckendahl J et al (2017) The addition of a sagittal image fusion improves the prostate cancer detection in a sensor-based MRI /ultrasound fusion guided targeted biopsy. BMC Urol 17(1):7

16. Günzel K, Haas M, Maxeiner A et al (2017) Predictive parameters identifying men eligible for a sole MRI/ultrasound fusion-guided targeted biopsy without an additional systematic biopsy. Urol Int 98(1):15-21

17. Maxeiner A, Kittner B, Blobel C et al (2018) Primary magnetic resonance imaging/ultrasonography fusion-guided biopsy of the prostate. BJU Int 122(2):211-218
18. Maxeiner A, Stephan C, Durmus T, Slowinski T, Cash H, Fischer T (2015) Added value of multiparametric ultrasonography in magnetic resonance imaging and ultrasonography fusion-guided biopsy of the prostate in patients with suspicion for prostate cancer. Urology 86(1):108-114

19. Epstein JI, Allsbrook WC Jr, Amin MB, Egevad LL, ISUP Grading Committee (2005) The 2005 International Society of Urological Pathology (ISUP) consensus conference on Gleason grading of prostatic carcinoma. Am J Surg Pathol 29:1228-1242

20. Leisenring W, Alonzo T, Pepe MS (2000) Comparisons of predictive values of binary medical diagnostic tests for paired designs. Biometrics 56:345-351

21. Faletti R, Battisti G, Discalzi A et al (2016) Can DW-MRI, with its $\mathrm{ADC}$ values, be a reliable predictor of biopsy outcome in patients with suspected prostate cancer? Abdom Radiol (NY) 41:926-933

22. Yoshimitsu K, Kiyoshima K, Irie H et al (2008) Usefulness of apparent diffusion coefficient map in diagnosing prostate carcinoma: correlation with stepwise histopathology. J Magn Reson Imaging 27:132-139

23. Lee H, Hwang SI, Lee HJ, Byun SS, Lee SE, Hong SK (2018) Diagnostic performance of diffusion-weighted imaging for prostate cancer: peripheral zone versus transition zone. PLoS One 13: e0199636

24. Girouin N, Mege-Lechevallier F, Tonina Senes A et al (2007) Prostate dynamic contrast-enhanced MRI with simple visual diagnostic criteria: is it reasonable? Eur Radiol 17:1498-1509

25. Chesnais AL, Niaf E, Bratan F et al (2013) Differentiation of transitional zone prostate cancer from benign hyperplasia nodules: evaluation of discriminant criteria at multiparametric MRI. Clin Radiol 68:e323-e330

26. Oto A, Kayhan A, Jiang Y et al (2010) Prostate cancer: differentiation of central gland cancer from benign prostatic hyperplasia by using diffusion-weighted and dynamic contrast-enhanced MR imaging. Radiology 257:715

27. Hoeks CM, Hambrock T, Yakar D et al (2013) Transition zone prostate cancer: detection and localization with 3-T multiparametric MR imaging. Radiology 266:207-217

28. Kim JH, Kim JK, Park BW, Kim N, Cho KS (2008) Apparent diffusion coefficient: prostate cancer versus noncancerous tissue according to anatomical region. J Magn Reson Imaging 28:11731179

29. Yoshizako T, Wada A, Hayashi T et al (2008) Usefulness of diffusion-weighted imaging and dynamic contrast-enhanced magnetic resonance imaging in the diagnosis of prostate transition-zone cancer. Acta Radiol 49:1207-1213

30. Jung SI, Donati OF, Vargas HA, Goldman D, Hricak H, Akin O (2013) Transition zone prostate cancer: incremental value of diffusion-weighted endorectal MR imaging in tumor detection and assessment of aggressiveness. Radiology 269:493-503

31. Bao J, Wang X, Hu C, Hou J, Dong F, Guo L (2017) Differentiation of prostate cancer lesions in the transition zone by diffusionweighted MRI. Eur J Radiol Open 4:123-128

32. Weiss B, Loeb S (2015) MRI/ultrasound fusion biopsy versus standard 12-Core biopsy. Rev Urol 17:113-115

33. Rouvière O, Puech P, Renard-Penna R et al (2019) Use of prostate systematic and targeted biopsy on the basis of multiparametric MRI in biopsy-naive patients (MRI-FIRST): a prospective, multicentre, paired diagnostic study. Lancet Oncol 20:100-109

34. Rosenkrantz AB, Ginocchio LA, Cornfeld D et al (2016) Interobserver reproducibility of the PI-RADS version 2 lexicon: a multicenter study of six experienced prostate radiologists. Radiology 280:793-804

Publisher's note Springer Nature remains neutral with regard to jurisdictional claims in published maps and institutional affiliations. 